\title{
Mechanisms of Immune Response to Intracerebral Allografts in the Model of Allograft Rejection Induced by Systemic Immunization with Donor Tissue
}

\author{
M. Poltorak, M. Isono, H. Kulaga, A.J. Adams and W.J. Freed \\ NIMH Neuroscience Center at St. Elizabeths, \\ Washington, D.C. 20032, USA
}

The characteristics of immune responses to neuronal tissue grafts within the CNS are still obscure. To investigate these properties, we chose the transplantation model in which immune responses against brain allografts are amplified in host peripheral lymphoid organs by systemic immunization with donor tissue. We studied several combinations of host and donor rat strains. Rejection responses varied considerably between groups. Systemic sensitization with BN-RT1n tissue results in the rejection of established BN-RT1n neuronal allografts in the lateral ventricle of host F344-RT1I rats (Freed et al., Prog Brain Res 1988; 78: 233-241). Surprisingly, if the host and donor strains are reversed, similar sensitization-induced brain graft rejection does not occur (Poltorak and Freed, Ann Neurol 1991; 29: 377-388). A similar asymmetry was observed in another experiment, when BN-RT1n donor grafts were severely rejected in LEW-RT1I hosts, while LEW-RT1I grafts in BN-RT1 hosts were not. Such results cannot be explained only in terms of genetic disparity between the donor and host strains. Despite complete genetic disparity, for some rat strain combinations, brain allografts can survive after systemic sensitization. Moreover, in the majority of strain combinations with the LEWRT1I as the donor strain, systemic sensitization did not produce complete graft destruction, whereas for all allografts from BN-RT1n donors, systemic sensitization induced severe immune responses in the host animals. Therefore, immunogenicity of the donor tissue is an important factor involved in neural graft rejection, but additional factors, including Ir gene, host MHC allele and host susceptibility to autoimmune disease, may also be important.
It has been suggested that grafted neuronal tissue demonstrates increased expression of MHC class I antigen, and that this enhancement is a crucial event leading to graft rejection. However, damage of the host brain during implantation procedures and injury of the grafted tissue itself also induces increased MHC class I antigen expression. Moreover, upregulation of MHC class II antigen expression in microglial cells, which can function as potential antigenpresenting cells, can be due to non-specific damage of host or donor tissue. Interestingly, we found that MHC class I immunoreactivity is usually more evident within grafts in the third ventricle, as compared to lateral ventricle. Increased expression of both MHC class I and II antigens in the grafted tissue did not predict allograft rejection. In certain rat strain combinations, allografts expressed increased MHC class I and II immunoreactivity without apparent immunological rejection responses and without lymphocytic infiltration. Therefore, it seems that simple upregulation of MHC antigen expression within the grafted tissue is not sufficient to induce a brain allograft rejection response.

After host sensitization with donor tissue, allografts showed increased cell density, as compared to sham-sensitized allografts. Nevertheless, in some rat strain combinations, we were not able to detect $T$ cell infiltration within the allografted tissue. In these cases, the infiltrating cells consisted primarily of activated microglial cells and blood-derived macrophages. These data support the hypothesis that some allografts do not induce T-cell mediated immune rejection responses. This type of reaction would be mediated primarily by microglial and macrophage cytokines. However, such immune responses are 
generally not sufficient to produce complete graft destruction. This immune response in host brain correlated with a lack of evident host spleen lymphocyte activation, as assessed by flow cytometry.

In other strain combinations, allograft rejection was mediated by $T$ cell populations. These rejected allografts showed enhanced MHC class I and II immunoreactivity similar to other nonrejected grafts. However, there was additionally a large influx of $T$ cell infiltration consisting of cytotoxic and helper lymphocytes. Activated microglial cells and macrophages were also present. The host animals with rejected allografts demonstrated full spleen lymphocyte activation with an increase in IL2R and CD4+ cells in the periphery as measured by flow cytometry. This type of immunological reaction, with $\mathrm{T}$ cell infiltration, was similar for most of the strongly rejected grafts. 

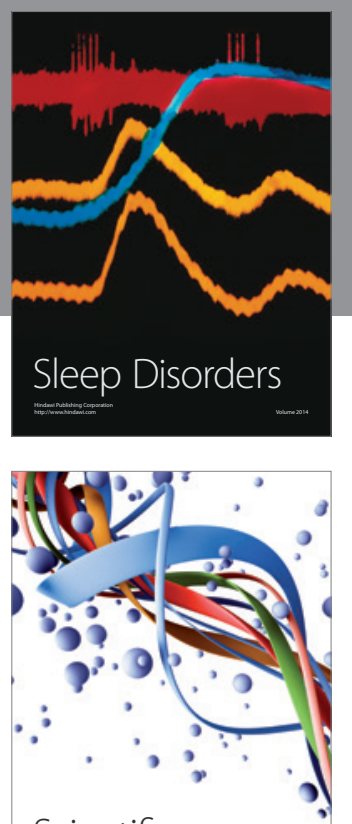

Scientifica
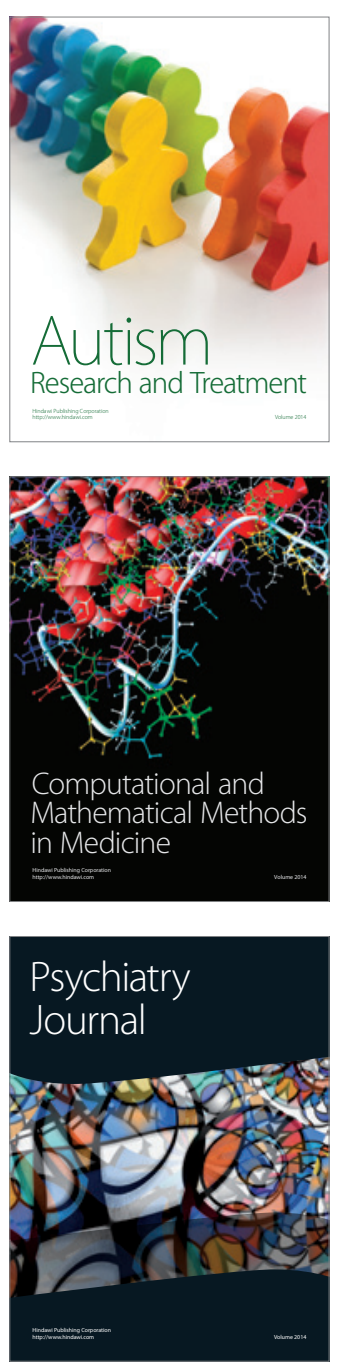
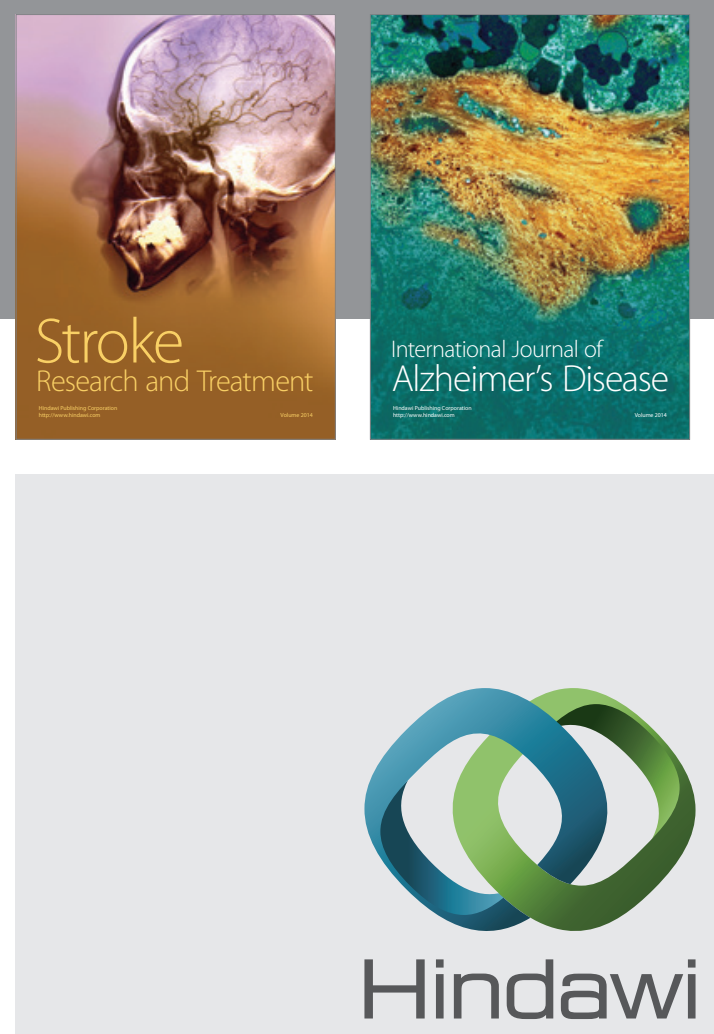

Submit your manuscripts at

http://www.hindawi.com
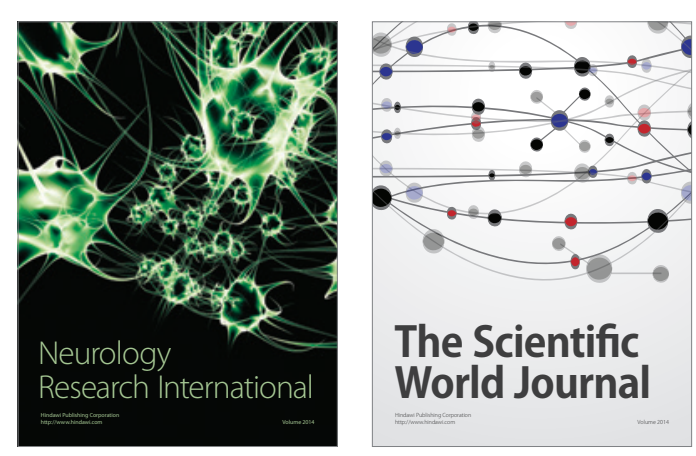

The Scientific World Journal

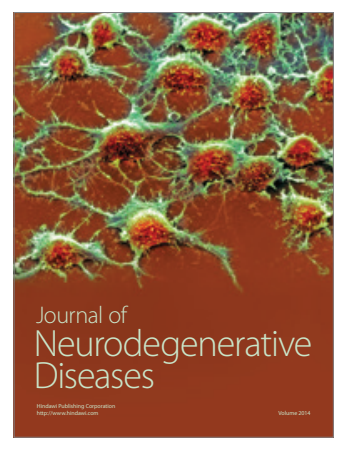

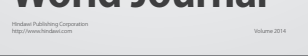

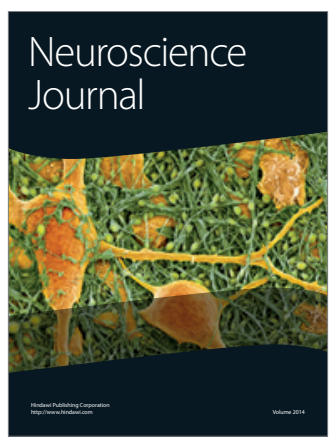

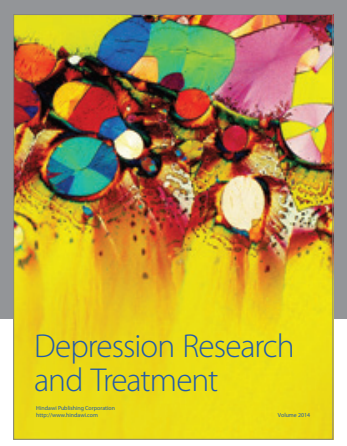
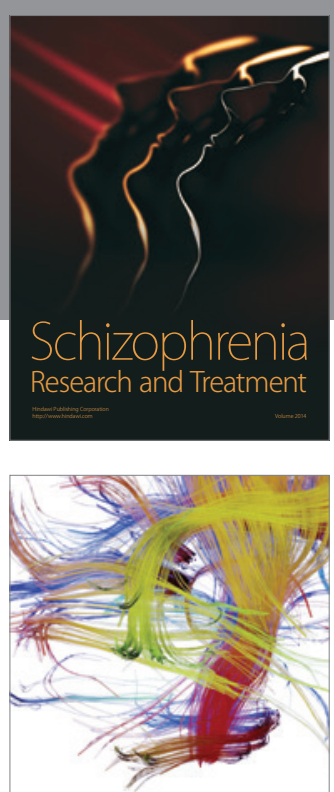

Brain Science

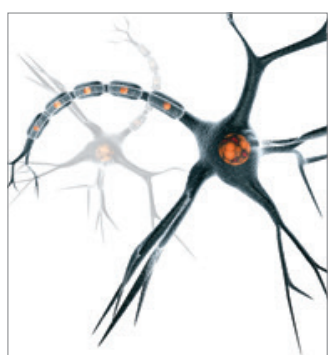

Neural Plasticity
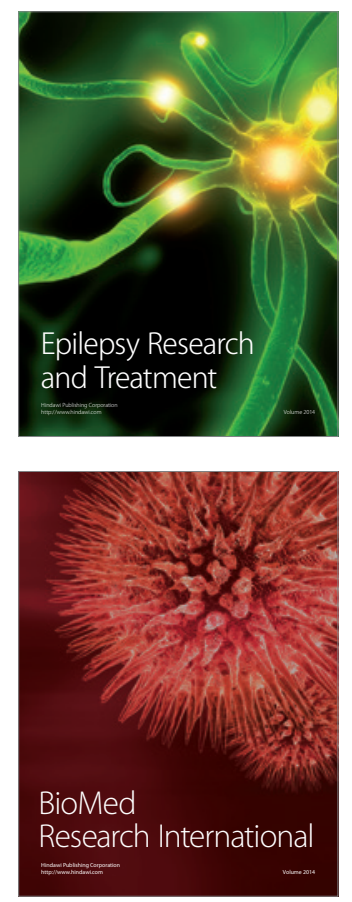

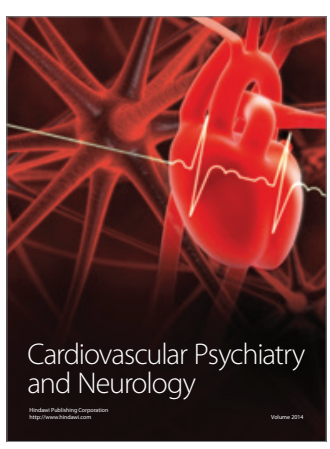

Parkinson's

Disease
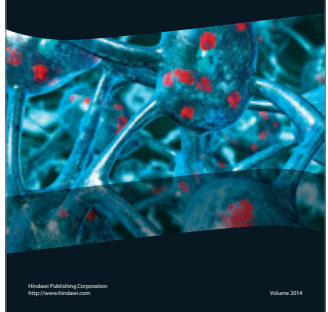